\title{
Intertrial interval effects in Pavlovian serial feature positive discriminations
}

\author{
PETER C. HOLLAND \\ Duke University, Durham, North Carolina
}

\begin{abstract}
The effects of the intertrial interval (ITI) on learning and performance in Pavlovian appetitive serial feature positive (SFP) discriminations were examined in three experiments with rats. With longer ITIs, acquisition was more rapid, and there was less transfer of the feature's behavioral control to a separately trained target cue, suggesting that longer ITIs encouraged the use of an occasion setting strategy. Behavior was also affected by discrimination-specific ITIs. Rats were trained with two SFP discriminations. The overall ITI was held constant, but the intervals between trials of one discrimination were varied by intermixing different numbers of trials from the other discrimination. Learning was more rapid when the intervals between trials of a single discrimination were longer. A sequential analyses showed that performance on a trial was impaired when it was preceded by a trial that included the same target cue but with the opposite trial outcome. The results are discussed in the frameworks of proactive interference effects and deletion-comparator processes (Cooper, Aronson, Balsam, \& Gibbon, 1990.)
\end{abstract}

Performance in learning tasks is often affected by the spacing of trials. Longer intertrial intervals (ITIs) encourage superior performance in a variety of tasks, including, for example, simple nondiscriminative Pavlovian conditioning (e.g., Gormezano \& Moore, 1969; Spence \& Norris, 1950), delayed matching-to-sample (e.g., Grant, 1975), and operant serial feature positive discrimination training (Holland, 1995). Moreover, many researchers have found that the ITI can affect within-trial interstimulus interval functions as well. For example, conditioning can occur with longer intervals between the onsets of the conditioned stimulus (CS) and unconditioned stimulus (US) if those CS-US pairings are separated by long ITIs than when they are massed in time (Gibbon, Baldock, Locurto, Gold, \& Terrace, 1977). In fact, Gibbon et al. and other investigators (e.g., Roberts \& Kraemer, 1982) have claimed that it is not the CS-US or intertrial intervals themselves that determines the amount of conditioning, but rather their ratio.

Holland (1995) examined the effects of ITI on the acquisition of operant serial feature positive discrimination learning, in which responding during a target cue was only reinforced when that target was preceded by another, feature cue (feature $\rightarrow$ target + / target - ). Acquisition of the discrimination was more rapid the longer the ITI. Furthermore, longer ITIs also seemed to favor the use of an occasion setting strategy (Holland, 1992) in solving that discrimination. That is, with shorter ITIs, the feature was

This research was supported in part by grants from the National Science Foundation and the National Institute of Mental Health. The author thanks Marie Crock, Corey McNamee, and John Chen for technical assistance. Correspondence should be addressed to P. C. Holland, Department of Psychology: Experimental, Duke University, Box 90086, Durham, NC 27708-0086 (e-mail: pch@duke.edu). more likely to exert direct control over responding, whereas, with longer ITIs, the feature cue apparently controlled conditioned responding by modulating the action of targetresponse-US associations. Finally, consistent with Gibbon et al.'s (1977) and Roberts and Kraemer's (1982) observations, the use of the occasion setting strategy was reasonably well predicted by the ratio of the ITI to the interval between the feature and target cues.

The experiments reported in this article examined the effects of the ITI on learning and performance in Pavlovian serial feature positive discriminations. Experiment 1 demonstrated the basic ITI effect that Holland (1995) observed with operant procedures (more rapid serial feature positive discrimination learning with longer ITIs), using Pavlovian procedures. Experiments 2 and 3 began the investigation of just what intervals are critical to this ITI effect in serial feature positive discrimination learning. In discrimination procedures, the average interval between all trials, between reinforced trials, between nonreinforced trials, between US presentations, and so forth, are all separately manipulable and might have different effects on learning and performance. For example, many accounts for ITI effects posit an important role for reinforcement (US presentation) rate (e.g., Gibbon \& Balsam; 1981). In simple nondiscriminative conditioning, the ITI and the interval between US presentations are typically the same. But Holland (1995) calculated the ITI in serial feature positive discrimination procedures on the basis of all trials, and so the relation between ITI and inter-US interval depended on the proportions of reinforced and nonreinforced trials. Experiments 2 and 3 attempted to disentangle the effects of overall ITI and interreinforcment interval from those specific to a particular serial feature positive discrimination by intermixing varying numbers of trials from another serial feature positive discrimination. 


\section{EXPERIMENT 1}

Experiment 1 examined Pavlovian serial feature positive discrimination learning with either short $(1-\mathrm{min})$ or long (8-min) ITIs, using a feature-target interval lengthy enough to discourage large amounts of simple featureUS conditioning $(20 \mathrm{sec})$. In an attempt to distinguish simple conditioning and occasion setting, the ability of the feature cue to produce conditioned responding during another, separately trained and then extinguished cue was examined in transfer tests. Other experiments from this laboratory show that simple conditioning, but not occasion setting, powers of a feature cue transfer readily to such separately trained target cues (Holland, 1992). Thus, occasion setting would be characterized by the observation of little transfer to the new target, and simple conditioning would be characterized by the observation of substantial transfer-that is, summation of the conditioned responding controlled by the feature and the new target.

\section{Method}

Subjects. Eight male and 8 female experimentally naive CDstrain rats (bred in a Duke Psychology Department breeding facility from Charles River stock), $120-180$ days old at the beginning of the experiment, served as subjects. The rats were individually housed in individual stainless steel cages in a vivarium that was maintained at $23^{\circ} \mathrm{C}$, with the lights on from 6:00 a.m. to 8:00 p.m. daily. All experimental sessions were carried out during the light portion of the cycle, between 8:00 a.m. and 11:00 a.m. The rats were maintained at $85 \%$ of their ad-lib weights by limiting their access to food; water was always available.

Apparatus. The apparatus consisted of eight individual chambers $(22.9 \times 20.3 \times 20.3 \mathrm{~cm})$ with aluminum front and back walls, clear acrylic sides and top, and a grid floor $(0.48-\mathrm{cm}$ stainless steel rods spaced $1.9 \mathrm{~cm}$ apart). A dimly illuminated food cup was recessed in the center of one end wall; a jeweled lamp (panel light) was located $5 \mathrm{~cm}$ above that recess. Each experimental chamber was enclosed in a sound-resistant shell with an acrylic window for viewing the rats. A speaker, used to present the auditory CSs, was mounted on the inside wall of the shell, $10 \mathrm{~cm}$ above and $10 \mathrm{~cm}$ to one side of the experimental chamber, even with the wall that contained the food cup. Ventilation fans provided masking noise $(70 \mathrm{~dB})$, and a 6-W, 110-V lamp (operated at $75 \mathrm{~V}$ ) behind a red lens opposite the speaker provided continuous dim background illumination. Two low-light television cameras were mounted $2.1 \mathrm{~m}$ from the experimental chambers, so each could include four chambers in its field of view. Videocassette recorders were programmed to record behaviors that occurred during the 10 -sec intervals before, during, and after CS presentations.

Procedure. Initially, all rats were trained to eat from the recessed food cup. In a single 16-min session, there were 16 presentations of the event used as the US throughout this experiment, two $45-\mathrm{mg}$ food pellets (Noyes) delivered $0.5 \mathrm{sec}$ apart, on a variable-time 1-min schedule.

The rats were then divided into two groups, each with 4 male and 4 female rats, and serial feature positive training was begun. Presentations of a serial compound of a 5-sec illumination of the panel light feature, followed after a 15 -sec empty interval by a 5 -sec presentation of an auditory target stimulus, were reinforced with food, and presentations of the 5-sec auditory target stimulus alone were not. The auditory stimulus was either a $1500-\mathrm{Hz}, 72-\mathrm{dB}$ tone or a 72-dB white noise, counterbalanced. In Group 1, the ITIs, measured from trial onset to trial onset, averaged $1 \mathrm{~min}$; in Group 8, they averaged $8 \mathrm{~min}$. The ITIs were randomly determined for each session, and they ranged from 0.5 to 1.5 times the mean ITI, rectangularly distributed. In both groups, each session comprised 16 trials; thus, the session duration was $16 \mathrm{~min}$ in Group 1 and $128 \mathrm{~min}$ in Group 8 . There were 8 reinforced compound trials and 8 nonreinforced targetalone trials in each of the first 10 sessions. For the next 10 sessions in Group 8 and the next 30 sessions in Group 1, there were 4 reinforced compound trials and 12 nonreinforced target-alone trials in each session.

Next, the cue to be used as the transfer target (either the noise or the tone, whichever was not used as the training target) was introduced into the training sessions. In the first 5 of these sessions, 4 of the nonreinforced target-alone trials were replaced with 5-sec reinforced presentations of the auditory transfer target cue; in the next 5 sessions, they were replaced by four 5 -sec nonreinforced presentations of that same cue. All rats then received a transfer test session to examine the effects of the panel light feature on responding to the separately trained and extinguished cue. In this session, there were three presentations of each of five types of trials: the original serial compound $(X \rightarrow 0)$, a transfer compound, comprising the 5sec panel light followed, after a 15 -sec empty interval, by the transfer target $(\mathrm{X} \rightarrow \mathrm{T})$, the original target alone $(\mathrm{O})$, the transfer target alone (T), and the feature (X) alone, followed by $20 \mathrm{sec}$ of empty measurement intervals so that responding could be assessed in the time period after the panel light when the target would normally occur. In each group, the ITI was held constant at the mean interval used in training for each group.

Behavioral observations. Behavioral observations were made from videotapes and were paced by auditory signals recorded on the tapes. Observations were made at 1.25 -sec intervals during the 5-sec period immediately prior to CS presentations, during the empty intervals on serial compound trials and during CS presentations. At each observation, one and only one behavior was recorded. The behavioral categories were defined so as to be mutually exclusive. For the test sessions, the rats were scored 1 at a time, such that there were four observations for each rat during each 5-sec interval. For the various training sessions, the observer shifted his/her gaze from rat to rat with each auditory signal, so that each rat was scored only once in each 5 -sec interval.

The primary mesasure of learning used in this experiment was food-directed behavior, which occurred in response to both visual and auditory CSs. Food-directed behavior included magazine behavior (standing motionless in front of the recessed food cup with the head or the nose within the recessed area), which was controlled by both visual and auditory CSs, and head jerk behavior (short, rapid horizontal and/or vertical movements of the head, often in or near the food cup), which was almost exclusively controlled by auditory CSs. In addition, rear behavior (standing on hind legs with front feet off the floor, and not grooming), specific to visual CSs, was recorded. These behaviors were described in detail by Holland (1977).

The index of frequency of each behavior was the percentage total observations, obtained by dividing the frequency of that behavior in any observation interval by the total number of observations made in that interval. Note that, because the rate of observations was constant within each observation interval, this measure is an absolute frequency measure, which compensates only for the duration of the observation interval. Two observers (M.C. and P.C.H.) scored the behavioral data reported in these experiments. All of the training session data were scored by M.C., and the test data were scored by both M.C. and P.C.H. When only decisions concerning the occurrence of food cup behavior, rear behavior, or neither were considered, the two observers agreed on over $95 \%$ of over 8,000 joint observations $(r=.99)$; when food cup behavior was subdivided into magazine and head jerk behaviors, agreement fell to $92 \%(r=.94)$.

Statistical analyses used the $p<.05$ level of significance; repeated measures analyses of variance (ANOVAs) used Greenhouse-Geisser corrected values. 


\section{Results}

Figure 1 shows behavior during the reinforced $(\mathrm{S}+)$ and nonreinforced $(\mathrm{S}-$ ) presentations of the target cues and during the pre-CS periods in the discrimination acquisition phase. The top panel shows the general class of food-directed behavior, the center panel shows head jerk behavior, and the middle panel shows magazine behavior. With all three measures, acquisition of the discrimination was more rapid in Group 8, in which the ITIs averaged
$8 \mathrm{~min}$, than in Group 1, in which the ITIs averaged $1 \mathrm{~min}$. Group $\times$ stimulus (reinforced or nonreinforced) $\times$ session ANOVAs of the data from the first 30 sessions (common to both groups) for each measure showed no overall effects of group $[F \mathrm{~s}(1,14)<1]$ but reliable group $\times$ stimulus and group $\times$ stimulus $\times$ session interactions $[F \mathrm{~s}(5,70) \geq 3.39]$.

Group 8's superior discrimination performance reflected both more responding on reinforced trials and less re-
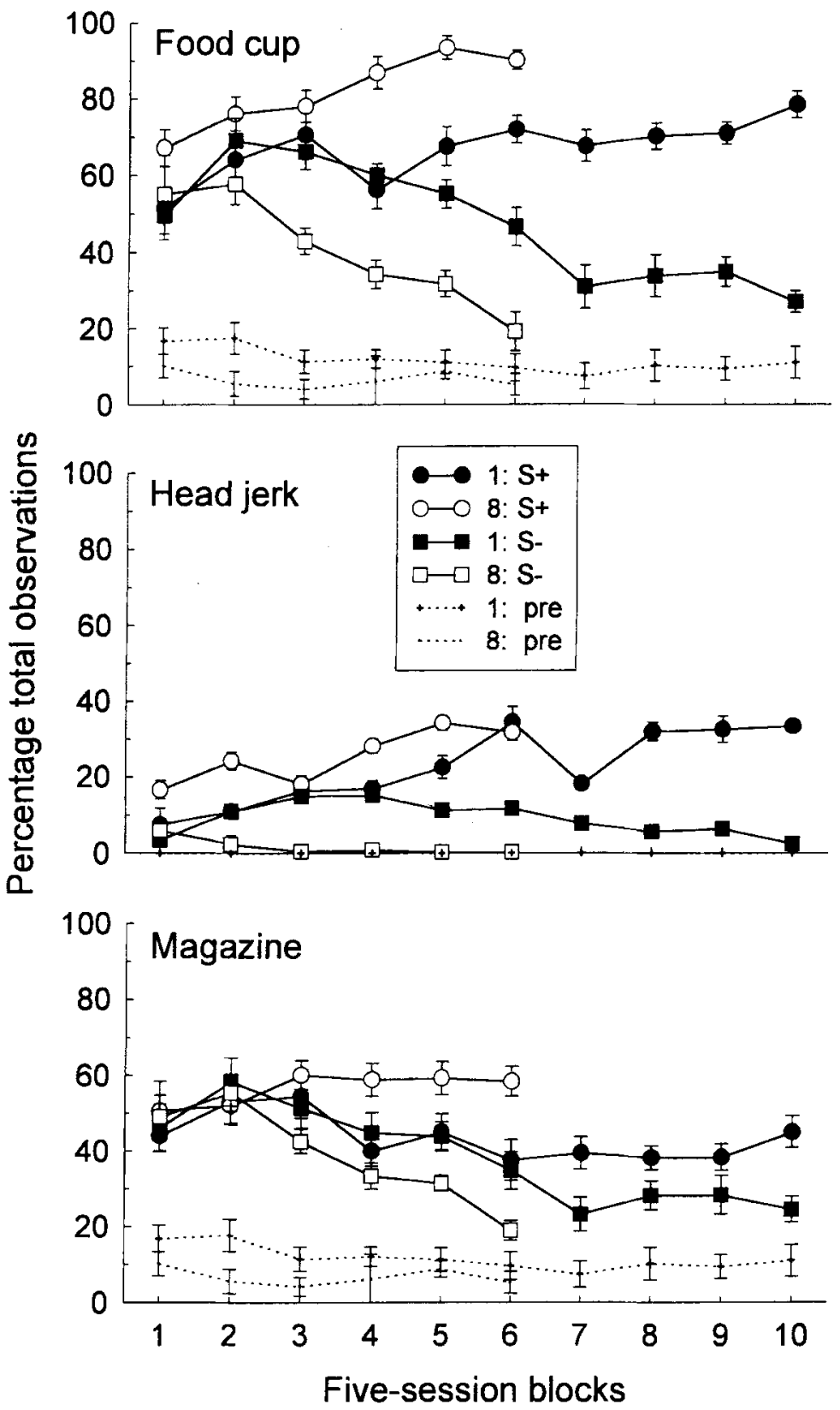

Figure 1. Acquisition (mean $\pm S E M$ ) of serial feature positive discriminations in Experiment 1.1 = Group 1, for which the intertrial intervals (ITIs) averaged $1 \mathrm{~min} ; 8=$ Group 8 , for which the ITIs averaged $8 \mathrm{~min} ; \mathrm{S}+=$ responding during the target stimulus on reinforced compound trials; $S-=$ responding on target-alone trials; pre $=$ responding during the prestimulus interval. 
sponding on nonreinforced trials than was observed in Group 1. ANOVAs of performance on reinforced trials alone showed reliable effects of group $[F \mathrm{~s}(1,14) \geq 6.85]$ and group $\times$ session interactions $[F \mathrm{~s}(5,70) \geq 3.18]$ for all three measures. Likewise, ANOVAs of performance on nonreinforced trials alone showed reliable effects of group $[F \mathrm{~s}(1,14) \geq 9.22]$ and group $\times$ session interactions $[F \mathrm{~s}(5,70) \geq 4.92]$ for food-directed and head jerk behaviors but not for magazine behavior $(F \mathrm{~s}=1.95$ and 1.61 , respectively).

In the transfer test, Group 8 maintained a high level of performance on the original discrimination but showed no evidence of transfer to the separately trained and extinguished target. In contrast, Group 1 displayed poorer performance on the original discrimination but greater transfer. Discrimination difference scores for food-directed behavior, computed by subtracting responding on targetalone trials from that during the target on compound trials, were $56.2 \% \pm 7.2 \%$ (mean $\pm S E M)$ for the original discrimination and $3.1 \% \pm 4.6 \%$ for the transfer discrimination in Group 8 and $35.4 \% \pm 10.2 \%$ and $18.8 \% \pm 7.8 \%$ in Group 1. A group $\times$ target (original or transfer) ANOVA of these difference scores showed a significant group $X$ target interaction $[F(1,14)=6.09]$. Analyses of simple effects showed that the difference scores were greater for the original than for the transfer discrimination in Group 8 $[F(1,14)=44.60]$, but not in Group $1[F(1,14)=1.11]$. Analyses of food-directed responding on individual trial types (Table 1) showed reliably more responding on feature $\rightarrow$ transfer target and feature-alone trials in Group 1 than in Group $8[F \mathrm{~s}(1,14) \geq 4.86]$, but no other betweengroups differences were reliable. (Head jerk and magazine behaviors considered individually each showed patterns similar to those of the combined food-directed behavior measure, but analyses such as those just described failed to reach significance.)

\section{Discussion}

The results of Experiment 1 extend Holland's (1995) results to Pavlovian procedures. As in Holland's experiments, the acquisition of serial feature positive discrimination performance was more rapid with longer ITIs. Also as in Holland's experiments, there was less transfer of the feature's behavioral control to a separately trained target cue after training with longer ITIs, suggesting that the use of longer ITIs encouraged the adoption of an occasion setting strategy.
One difference between the results of Experiment 1 and those of Holland (1995) deserves comment. In Experiment 1 , the superior performance of the rats trained with longer ITIs was revealed both in less responding on nonreinforced target-alone trials and in more responding on reinforced feature-target compound trials. Holland found no effects of ITI on responding on reinforced trials, which was somewhat surprising, given the extensive literature of ITI effects with simple, nondiscriminative conditioning (reviewed in the introduction of this paper). However, prior to discrimination training with different ITIs, all of Holland's rats received extensive pretraining of responding to another cue with a common ITI; generalization between that cue and the same-modality discrimination target cue may have masked ITI-dependent differences in acquisition. No such pretraining was given in the present experiment. Furthermore, in Holland's study, it is possible that the costs of delayed responding (reinforcement was delivered immediately after the first response to the target cue) were sufficient to overwhelm ITI effects. In the present experiment, which used Pavlovian procedures, performance of the CR during the CS did not affect the timing of US delivery. Thus, it seems likely that Holland's failure to find ITI effects on responding to the reinforced CS was more the result of these (or other) procedural details than an overall insensitivity of conditioning to ITI.

\section{EXPERIMENT 2}

The use of shorter ITIs in serial feature positive discrimination learning increases the presentation rate of the US, the feature, the target, reinforced trials, nonreinforced trials, and trials in general, and it increases the proximity of any two types of trials. Any of these consequences of massed trials might contribute to the ITI effects observed in Experiment 1 and in Holland's (1995) experiments. Experiment 2 examined the effects of intermixing trials from one serial feature positive discrimination ("A"; $X \rightarrow A+, A-$ ) with trials from another such discrimination (" $\mathrm{B}$ "; $\mathrm{Y} \rightarrow \mathrm{B}+, \mathrm{B}-$ ). Intermixing various proportions of $A$ and $B$ discrimination trials within each session in different groups of rats, but holding the session duration and the total number of trials constant, permitted examination of the effects of variation in the intervals between trials of one discrimination while the overall ITI was unchanged. The primary question ad-

Table 1

Transfer Test in Experiment 1

\begin{tabular}{|c|c|c|c|c|c|c|c|c|c|c|c|c|}
\hline \multirow[b]{2}{*}{ Group } & \multicolumn{2}{|c|}{$\mathrm{F} \rightarrow \mathrm{O}$} & \multicolumn{2}{|c|}{$\mathrm{O}$} & \multicolumn{2}{|c|}{$\mathrm{F} \rightarrow \mathrm{T}$} & \multicolumn{2}{|r|}{$\mathrm{T}$} & \multicolumn{2}{|c|}{$F$} & \multicolumn{2}{|c|}{ Prestimulus } \\
\hline & $M$ & $S E M$ & $M$ & $S E M$ & $M$ & $S E M$ & $M$ & $\overline{S E M}$ & $M$ & $\overline{S E M}$ & $M$ & $S E M$ \\
\hline 1 & 63.5 & \pm 10.0 & 28.1 & \pm 6.6 & 54.2 & \pm 7.4 & 35.4 & \pm 7.2 & 37.5 & \pm 7.7 & 8.0 & \pm 6.1 \\
\hline 8 & 71.9 & \pm 5.5 & 15.6 & \pm 3.3 & 35.4 & \pm 5.0 & 32.3 & \pm 3.1 & 16.7 & \pm 3.3 & 8.0 & \pm 4.3 \\
\hline
\end{tabular}

Note-Entries are mean $\pm S E M$ food cup behavior, as a percentage of all observations. $\mathbf{F}=$ feature stimulus; $\mathrm{O}=$ original target stimulus; $\mathrm{T}=$ transfer target stimulus. 
dressed in Experiment 2 was whether performance on a particular discrimination would more closely reflect the average interval between trials from that discrimination or the average overall ITI-that is, the interval between trials of any sort.

In Groups 1-1, 2-2, and 4-1.3, 16 trials were presented in each 16 -min session, so the overall ITI was $1 \mathrm{~min}$. (The group designations indicate the average interval between A trials and that between B trials, in minutes.) In Group 1-1, A and B trials were delivered in separate sessions, so that the interval between A trials and the interval between $B$ trials within sessions were also each $1 \mathrm{~min}$. In Group 2-2, there were $8 \mathrm{~A}$ and $8 \mathrm{~B}$ trials in each session, so that the inter- $A$ and inter-B intervals were both $2 \mathrm{~min}$. In Group 4-1.3, there were $4 \mathrm{~A}$ and $12 \mathrm{~B}$ trials, so that the inter-A intervals averaged $4 \mathrm{~min}$ and the inter-B intervals averaged $1.33 \mathrm{~min}$. Thus, these three groups all had the same overall ITI ( $1 \mathrm{~min}$ ) but different inter-A and inter-B intervals $(1,2$, or $4 \mathrm{~min}$, or 1,2 , or $1.3 \mathrm{~min}$, respectively). Finally, in Group 4-4, A and B trials were delivered in separate sessions, at the rate of 4 in each 16-min session. Thus, Groups 4-4 and 4-1.3 both had 4-min interA intervals but very different overall ITIs ( 4 or $1 \mathrm{~min}$ ).

\section{Method}

Subjects. Thirty-two experimentally naive female CD-strain rats (obtained from Charles River, Raleigh, NC), 90 days old at the beginning of the experiment, served as subjects. The rats were housed and maintained as in Experiment 1.

Apparatus. The apparatus was a set of eight chambers similar to those used in Experiment 1, except (1) the recessed food cup was $2 \mathrm{~cm}$ to the right of center, (2) there were infrared photocells (sampled by computer circuitry at approximately $1 \mathrm{KHz}$ ) flush with the recessed food cup, (3) there was another 6-W lamp (houselight) mounted next to the speaker, (4) there was no background illumination, and (5) there were no television cameras.

Procedure. Initially, all rats were trained to eat from the recessed food cup. In a single 16-min session, there were 16 presentations of the event used as the US throughout this experiment, the delivery of $0.3 \mathrm{ml}$ of $0.2 \mathrm{M}$ sucrose solution, on a variable-time 1 -min schedule.

Simple conditioning. All rats then received four 16-min sessions of simple conditioning with the two serial compounds to be used later in discrimination training. Each serial compound comprised a 5-sec visual stimulus ( $\mathrm{X}$ or $\mathrm{Y}$ ) followed, after a 15 -sec empty interval, by a 5-sec auditory stimulus ( $A$ or $B$ ), reinforced immediately with sucrose. The visual cues used were a flashing $(5 \mathrm{~Hz})$ of the panel light and steady illumination of the houselight, and the auditory cues used were an intermittent white noise $(75 \mathrm{~dB})$ and a steady $1500-\mathrm{Hz}$ tone $(78 \mathrm{~dB})$. All four combinations of the four events were equally represented within each group; for each rat, one compound was designated $\mathrm{X} \rightarrow \mathrm{A}$, and the other was designated $\mathrm{Y} \rightarrow \mathrm{B}$. Group 1-1 received 16 reinforced $X \rightarrow A$ trials in the first and fourth sessions of this phase and 16 reinforced $\mathrm{Y} \rightarrow \mathrm{B}$ trials in the second and third sessions. Group 4-4 received 4 reinforced $X \rightarrow A$ trials in the first and fourth sessions of this phase and 4 reinforced $Y \rightarrow B$ trials in the second and third sessions. Group 2-2 received 8 reinforced $\mathrm{X} \rightarrow \mathrm{A}$ trials and 8 reinforced $\mathrm{Y} \rightarrow \mathrm{B}$ trials in each of the four sessions. Group 4-1.3 received 4 reinforced $X \rightarrow A$ trials and 12 reinforced $\mathrm{Y} \rightarrow \mathrm{B}$ trials in each of the sessions. In Group 4-4, the ITIs averaged $4 \mathrm{~min}$, but, in the remaining groups, the ITIs averaged $1 \mathrm{~min}$.

Serial feature positive discrimination training. Next, the rats received training on the two serial feature positive discriminations,
" $A$ " and "B." Each discrimination cycle comprised one reinforced serial compound trial $(\mathrm{X} \rightarrow \mathrm{A}+$ or $\mathrm{Y} \rightarrow \mathrm{B}+)$ and three nonieinforced 5-sec target-alone trials $(\mathrm{A}-$ or $\mathrm{B}-$ ). In each 16-min session, the rats in Group 4-4 received a single discrimination cycle with either the A or the B discrimination, the rats in Group 1-1 received four cycles of either the A or the B discrimination, the rats in Group 2-2 received two cycles of each discrimination, and the rats in Group 4-1.3 received one cycle of the A discrimination and three cycles of the B discrimination. In Group 4-4, the overall ITIs, measured from trial onset to trial onset, averaged $4 \mathrm{~min}$, and in the remaining groups they averaged $1 \mathrm{~min}$. The ITIs were randomly determined for each session and ranged from 0.5 to 1.5 times the mean ITI, rectangularly distributed. Groups 1-1 and 4-4 received 16 sessions with the A discrimination and 16 sessions with the $B$ discrimination, randomly intermixed. In Groups 2-2 and 4-1.3, each of the 32 sessions included trials with both the $\mathrm{A}$ and the $\mathrm{B}$ discrimination.

Data analysis. The primary measure of conditioning was the percentage of time during the CS that the photocell in the food cup was activated. (The rate of food cup entry and the latency to the first food cup entry after CS onset were also recorded, but not reported.) A simple measure of discrimination performance was constructed by subtracting performance during the nonreinforced target $\mathrm{CS}$ from performance during the reinforced CS.

\section{Results and Discussion}

Figure 2 shows the acquisition difference scores, as a function of the number of training cycles with each discrimination task (each cycle comprised one reinforced serial compound trial and three nonreinforced target-alone trials). For convenience, in Groups 1-1, 2-2, and 4-4, performance on the arbitrarily designated $\mathrm{A}$ and $\mathrm{B}$ discriminations is combined (ANOVAs showed no differences in performance on the $A$ and $B$ discriminations in any of these groups). The points labeled 1.3-4 refer to performance on the B discrimination in Group 4-1.3 (for which the average interval between those $B$ trials was $1.33 \mathrm{~min}$ ), and the points labeled 4-1.3 refer to performance on the A discrimination in that group (for which the average interval was $4 \mathrm{~min}$ ). Consistent with previous data (e.g., Experiment 1; Holland, 1995), acquisition of both the A and the B discrimination was more rapid in Group 4-4, in which both the overall ITI and the interval between trials from that discrimination were $4 \mathrm{~min}$, than in Groups 1-1 and 2-2, in which the overall ITIs were $1 \mathrm{~min}$ and the intervals between $A$ trials were 1 and 2 min, respectively.

More interesting is the performance of Group 4-1.3, which had overall ITIs of 1 min but inter-A intervals of $4 \mathrm{~min}$ and inter-B intervals of $1.33 \mathrm{~min}$. First, that group's performance on the A discrimination was considerably better than its performance on the $\mathrm{B}$ discrimination. Second, although that group's performance on the A discrimination was not as good as that in Group 4-4 (which had both 4-min ITIs and 4-min inter-A intervals), it was superior to performance in Groups 1-1 and 2-2, which, like Group 4-1.3, had 1-min ITIs overall but had 1-min and 2-min inter-A intervals, respectively. Third, Group 41.3 's performance on the $\mathrm{B}$ discrimination was poorer than Group 2-2's performance on that discrimination. Thus, not only did the overall ITI play a significant role (as indicated by the superiority of performance in Group 4-4) 


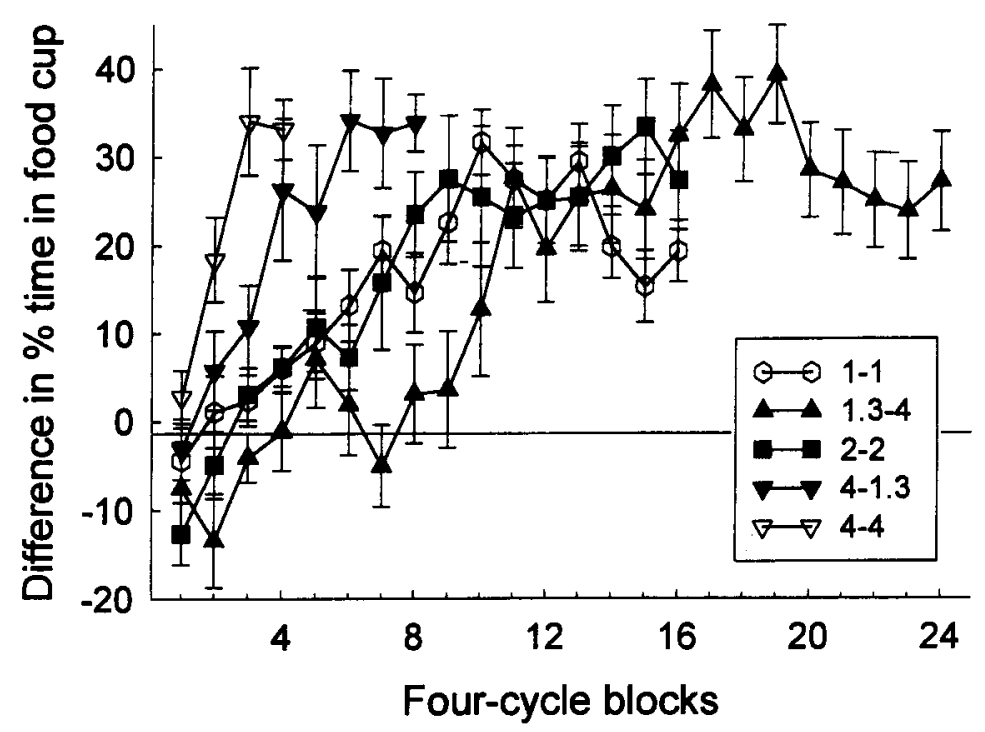

Figure 2. Acquisition (mean $\pm S E M$ ) of discriminative responding (responding on reinforced compound trials minus responding on nonreinforced targetalone trials) in Experiment 2, plotted by cycles. Each cycle comprised 1 reinforced compound trial and 3 nonreinforced target-alone trials. See text for explanation of the group designations in the legend.

but also performance was substantially influenced by the interval between trials on a particular discrimination when the overall ITI was constant.

These claims were supported by a variety of statistical analyses. First, a discrimination (A or B) $\times$ session block ANOVA of the data from Group 4-1.3 over the first 8 four-cycle blocks (all of those that included both discriminations) showed a reliable difference between performance on the $\mathrm{A}$ and $\mathrm{B}$ discriminations $[F(1,7)=$ 24.18]. Second, separate group $\times$ session ANOVAs of the difference scores for the $\mathrm{A}$ and $\mathrm{B}$ discriminations were conducted over the first 4 four-cycle blocks (common to all groups), the first 8 four-cycle blocks (common to all groups except Group 4-4), and the first 16 four-cycle blocks (common to Groups 1-1, 2-2, and, for the B discrimination only, 4-1.3). ANOVAs over the first 4 four-cycle blocks showed reliable effects of group for both the $\mathrm{A}$ and the $\mathrm{B}$ discrimination $\left[F_{\mathrm{s}}(3,28)=12.58\right.$ and 23.89, respectively]. Tukey HSD tests showed these discrimination difference scores for the A discrimination to be reliably greater in Group 4-4 than the scores in Group 4-1.3, which in turn were reliably greater than the scores in Groups 1-1 and 2-2, which did not differ. Tukey HSD tests for the B discrimination showed performance in Group 4-4 to be superior to that in any of the other groups, which did not differ. ANOVAs over the first 8 fourcycle blocks also showed reliable effects of group for both the $\mathrm{A}$ and the $\mathrm{B}$ discrimination $\left[F_{\mathrm{s}}(2,21)=6.65\right.$ and 6.41 , respectively]. Tukey HSD tests showed performance on the A discrimination to be reliably better in Group 4-1.3 than in Groups 1-1 and 2-2, which did not differ, and performance on the $B$ discrimination to be reliably poorer in Group 4-1.3 than in Groups 1-1 and 2-2, which did not differ. ANOVAs over the first 16 four-cycle blocks showed no reliable effect of group for either the A $[F(1,14)<1]$ or the $\mathrm{B}[F(2,21)=2.53]$ discrimination.

Finally, one-way ANOVAs showed that the number of cycles to reach a criterion of $20 \%$ difference between S+ and $\mathrm{S}-$ responding in three consecutive cycles differed reliably as a function of group for both the $\mathrm{A}$ and the $\mathrm{B}$ discrimination $[F \mathrm{~s}(3,28)=11.34$ and 9.11 , respectively]. Subsequent Tukey HSD tests showed that, for the A discrimination, criterion was reached significantly faster in Groups 4-4 (mean of 8.0 cycles) and 4-1.3 (19.1 cycles) than in Groups 1-1 (33.1 cycles) and 2-2 (41.2 cycles), but neither of those pairs of groups differed among themselves. For the B discrimination, Group 4-4 (8.1 cycles) reached criterion faster than any of the other groups, which did not differ reliably among themselves (range of $35.8-43.2$ cycles).

Comparable analyses of performance to S+ and Sindividually (data not shown) were also carried out. All of the trends described previously for the difference score measures were apparent in responding to $\mathrm{S}-$ alone (with a lower percentage time in the food cup corresponding to higher difference scores), although several of those effects did not reach statistical significance. Many of those trends were also apparent in responding to $\mathrm{S}+$ (with a higher percentage time in the food cup corresponding to higher difference scores), but few were statistically reliable.

As just presented, these data are generally consistent with roles for both overall ITI and discriminationspecific ITIs. The superiority of performance in Group 4-4 
shows that longer overall ITIs generated better learning, even when the discrimination-specific ITI was equated (compare with the A discrimination in Group 4-1.3). Furthermore, the ordering of performance in Groups 2-2 and 4-1.3 (both of which had the same overall ITIs, $1 \mathrm{~min}$ ) is consistent with a role for discrimination-specific interval as well: Performance was best with 4-min inter-A intervals (A in Group 4-1.3), intermediate with 2-min inter-A intervals (Group 2-2), and worst with 1.3-min intervals (B in Group 4-1.3).

However, the performance of Group 1-1 suggests other interpretations of these data. Because the discriminationspecific intervals in Group 1-1 were $1 \mathrm{~min}$, performance in that group should have been worst of all, rather than intermediate, as observed. Several accounts for this discrepancy come to mind. Two preserve the notion that discrimination-specific interval is important. First, discrimination performance might be insensitive to the difference between 1-min (Group 1-1) and 2-min (Group 2-2) discrimination-specific intervals, and differential performance in Group 4-1.3 might be the result of a contrast effect, which made the $A$ (4-min interval) discrimination performance better and the $\mathrm{B}(1.3$-min interval) discrimination performance worse than would have occurred if each group had only one interval value for both discriminations. Second, Group 1-1 might have shown enhanced performance relative to Group 2-2 because of some unspecified advantage of having to deal with only one discrimination in each session.

Two other accounts for Group 1-1's intermediate performance deny the importance of discrimination-specific interval. Both are based on the fact that, in Experiment 2, the number of cycles in each session differed across the various groups. Consequently, between-groups compar- isons of performance over equal numbers of cycles may include data from different numbers of sessions and after different amounts of training of the other discrimination. Some investigators have noted that learning proceeds more rapidly when fewer trials are given in each session, independent of ITI (e.g., Papini \& Dudley, 1993). Thus, in Group 4-1.3, for example, performance on the A discrimination may have been superior to performance on the $B$ discrimination not because of an advantage in trial spacing per se but because of some other advantage of having fewer $A$ trials than $B$ trials in each session.

Another perhaps even simpler possibility is to consider the effects of generalization between the two discriminations, A and B. Suppose the rats generalized completely, treating the A and B discriminations as one. In that case, the appropriate comparisons would not be of the number of cycles of a particular discrimination but rather the total number of cycles of all discriminations, which was identical in all groups except Group 4-4.

Figure 3 shows the data from the acquisition phase of Experiment 2, replotted as a function of blocks of sessions rather than blocks of cycles. ANOVAs such as those conducted for the cycles data revealed no reliable effects, except for sessions and a superiority of performance in Group 4-4 to that in Group 1-1 (Tukey HSD test).

The superiority of performance in Group 4-4 to that in Group 1-1 shows that the advantage of the 4-min ITI over the 1-min ITI (or of having one fourth as many trials in each session) was large enough to overcome the former group's disadvantage of receiving one fourth as many training trials over the same number of sessions. By contrast, the lack of differences among performances in the remaining conditions is consistent with the possibility that the rats in the remaining groups failed to dis-

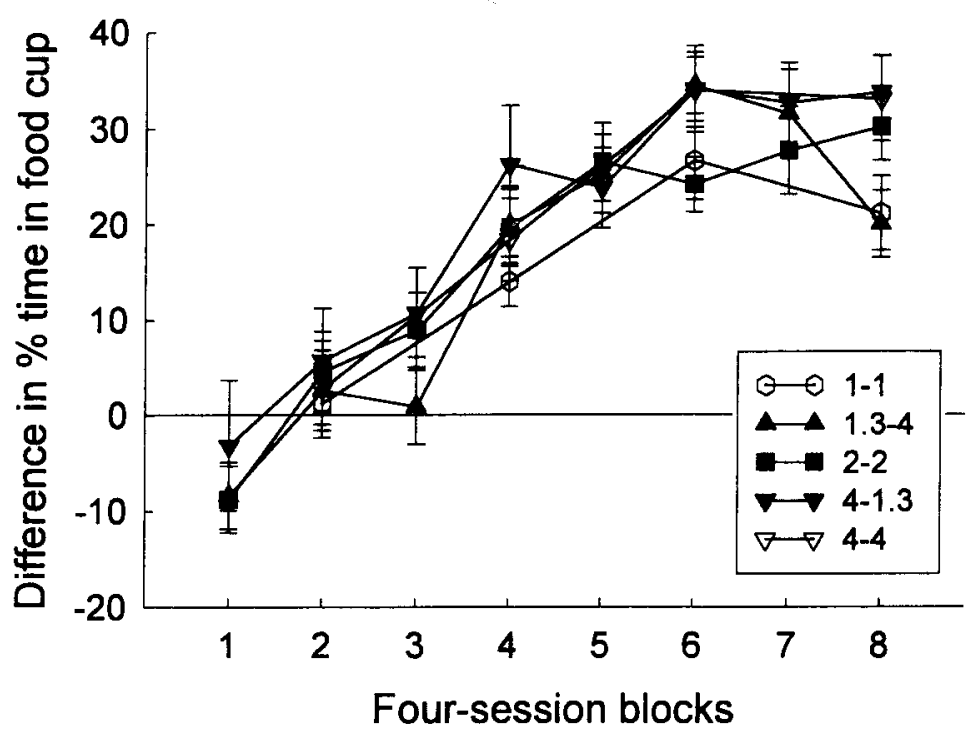

Figure 3. Acquisition (mean $\pm S E M$ ) of discriminative responding (responding on reinforced compound trials minus responding on nonreinforced targetalone trials) in Experiment 2, plotted by sessions. See text for explanation of the group designations in the legend. 
tinguish between trials on the A and B discriminations. On the other hand, previous experiments with these same stimuli showed that the two serial compounds are readily discriminated (e.g., Holland, 1989a, 1989b). In the absence of complete generalization between the $A$ and $B$ discriminations, the observation that all groups trained with overall ITIs of $1 \mathrm{~min}$ acquired in the same number of sessions implies that the advantage of using longer intervals between trials from the same discrimination might have been large enough to overcome the disadvantage of giving fewer training cycles with those intervals in each session. Experiment 3 was designed to disentangle the effects of discrimination-specific ITI and number of cycles per session, which were confounded in Experiment 2, and to reduce the possible contribution of generalization between the two discriminations.

\section{EXPERIMENT 3}

In Experiment 3, rats again received training cycles with two discriminations, A and B. For all these groups, the overall ITI was $1 \mathrm{~min}$, but the interval between A trials varied across the groups: 2,3 , or $4 \mathrm{~min}$. In each session, each group received only one cycle of the A discrimination but varying numbers of cycles of the $B$ discrimination. Thus, the interval between $A$ trials and the number of A cycles in each session were not confounded, as in Experiment 2 . To minimize the contribution of generalization between the A and B discriminations, the modalities of the features and targets differed across discriminations.
Experiment 3 also considered whether the inter- $\mathrm{A}$ interval effects observed reflected variations in learning or in performance. At the end of training, all rats were tested with the longest and shortest inter- $A$ intervals, keeping the overall ITI constant by interpolating different numbers of trials from the B discrimination. Finally, in an effort to more completely describe the ITI effects observed, the data from this test session were examined for sequence effects. Many investigators have noted that performance on individual trials in discrimination tasks can be affected by the nature of the immediately preceding trial (e.g., Grant, 1975; Han, Gallagher, \& Holland, 1998; Hogan, Edwards, \& Zentall, 1981; Pontecorvo, 1983) or by the occurrence of a response that was incorrect for the present trial (e.g., Roitblat \& Scopatz, 1983), especially when the ITIs are relatively brief.

\section{Method}

Subjects and Apparatus. Twenty-four experimentally naive female CD-strain rats (obtained from Charles River, Raleigh, NC), 90 days old at the beginning of the experiment, served as subjects. The rats were housed and maintained as in Experiments 1 and 2. The apparatus was that used in Experiment 2.

Procedure. Initially, all rats were trained to eat from the recessed food cup, as in Experiment 2. All rats then received four sessions of simple conditioning, with the two serial compounds to be used later in discrimination training. Each $\mathrm{X} \rightarrow \mathrm{A}$ compound comprised a 5-sec flashing $(5 \mathrm{~Hz})$ of the panel light followed, after a $15-\mathrm{sec}$ empty interval, by a 5 -sec steady $1500-\mathrm{Hz}$ tone, reinforced immediately with sucrose; each $\mathrm{Y} \rightarrow \mathrm{B}$ compound comprised a 5-sec intermittent white noise followed, after a 15 -sec empty interval, by a steady illumination of the houselight also reinforced immediately

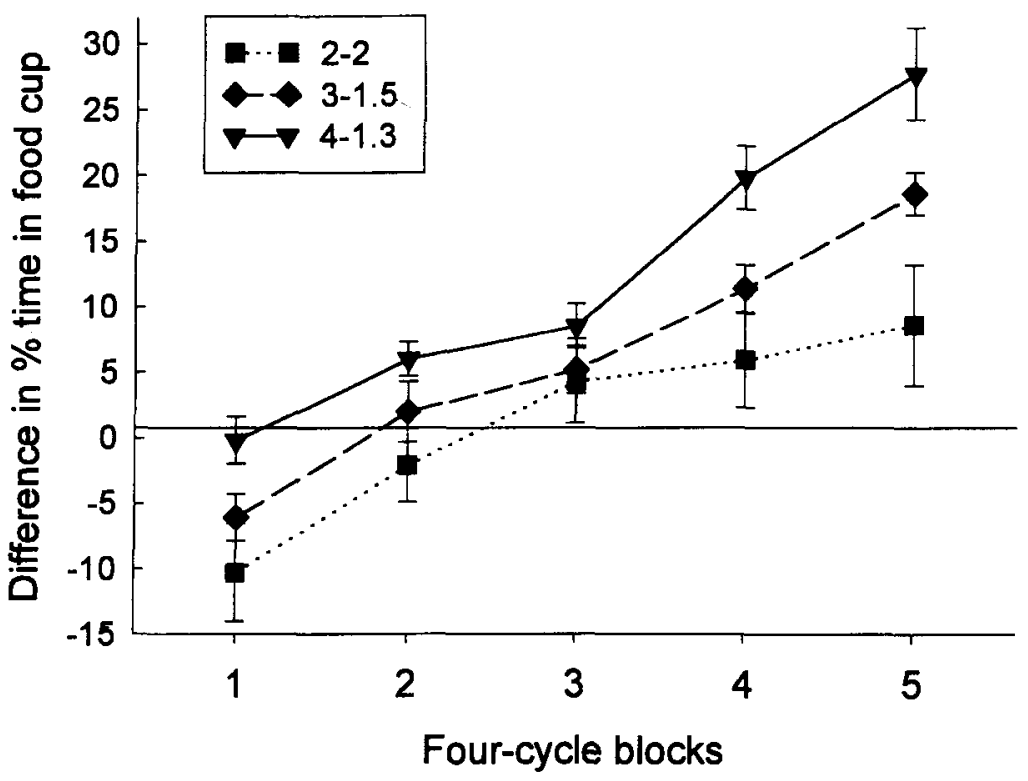

Figure 4. Acquisition (mean $\pm S E M$ ) of discriminative responding (responding on reinforced compound trials minus responding on nonreinforced target-alone trials) for the A discrimination in Experiment 3, plotted by cycles. Each cycle comprised 1 reinforced compound trial and 3 nonreinforced target-alone trials. See text for explanation of the group designations in the legend. 
with sucrose. Group 2-2 received 4 reinforced $X \rightarrow A$ trials and 4 reinforced $\mathrm{Y} \rightarrow \mathrm{B}$ trials in each of four 8-min sessions. Group 3-1.5 received 4 reinforced $X \rightarrow A$ trials and 8 reinforced $Y \rightarrow B$ trials in each of four $12-\mathrm{min}$ sessions. Group $4-1.3$ received 4 reinforced $X \rightarrow A$ trials and 12 reinforced $Y \rightarrow B$ trials in each of four $16-\mathrm{min}$ sessions. Thus, the average overall ITI was $1 \mathrm{~min}$ (distributed as in the previous experiments), whereas the average interval between $\mathrm{X} \rightarrow \mathrm{A}$ trials was 2,3 , or $4 \mathrm{~min}$ in the three groups.

Next, the rats received training on the two serial feature positive discriminations, "A" and "B." Each discrimination cycle comprised one reinforced serial compound trial and three nonreinforced 5-sec target-alone trials. In each of 20 sessions, the rats in all groups received a single cycle of the A discrimination and one, two, or three cycles of the B discrimination (Groups 2-2, 3-1.5, and 4-1.3, respectively). Session lengths and ITIs were the same as in the previous phase.

Finally, all rats' performance on the two discriminations under both the 2-2 and the 4-1.3 interval condition was examined in four test sessions. In the first and fourth sessions, identical to the training sessions received by Group 2-2, half of the rats in each group were tested with 2-min inter- $A$ and inter-B intervals. Each 8-min session included 1 reinforced $X \rightarrow A$ trial and 3 nonreinforced $A$ trials, intermixed with 1 reinforced $Y \rightarrow B$ trial and 3 nonreinforced $B$ trials. The other half of the rats in each group were tested with 4-min inter-A and 1.3-min inter-B intervals, identical to the training sessions received by Group 4-1.3. Each 16-min session included 1 reinforced $X \rightarrow A$ trial and 3 nonreinforced $A$ trials, intermixed with 3 reinforced $\mathrm{Y} \rightarrow \mathrm{B}$ trials and 9 nonreinforced $\mathrm{B}$ trials. In the second and third test sessions, the rats were tested with the other set of intervals. In all of the test sessions, the interval between each trial was fixed ( $1 \mathrm{~min}$, onset to onset). For the 2-min interval test, in one session, the trial sequence was $\mathrm{B}, \mathrm{YB}, \mathrm{A}, \mathrm{A}, \mathrm{B}, \mathrm{XA}, \mathrm{A}$, $B$; in the other session, Trials 4 and 5 were exchanged. These sequences were chosen to permit the analysis of the effects of $n-1$ trial type on performance on trial $n$ A discrimination trials (XA and A trials). For the 4/1.3-min interval test, in one session, the trial sequence was B, B, A, YB, B, A, B, XA, YB, B, B, A, B, YB, B, B; in the other session, Trials 5 and 9 were exchanged. These sequences were chosen to permit analysis of the effects of $n-1$ trial type on performance on trial $n \mathrm{~B}$ discrimination trials (YB and $\mathrm{B}$ trials).

\section{Results and Discussion}

Figure 4 shows the primary data from Experiment 3, the acquisition of the A discrimination. With an overall ITI of $1 \mathrm{~min}$ and one A discrimination cycle per session in each group, performance on the A discrimination was greater the longer the interval between A trials. Thus, the major finding of Experiment 2 was replicated in Experiment 3 under conditions in which it was less likely that either stimulus generalization or the number of $\mathrm{A}$ discrimination trials per session were major contributors. A group $\times$ block ANOVA showed reliable main effects of group $[F(2,21)=4.39]$ and session block $[F(4,84)=$ $151.98]$ and a significant group $\times$ session block interaction $[F(8,84)=5.02]$. A trend analysis showed reliable linear variation (across groups) in the linear trend over session blocks $[F(1,21)=13.62$; residual $F<1]$.

The left side of Figure 5 shows acquisition of the B discrimination, plotted as a function of discrimination cycles. As with the A discrimination, over the earlier portions of training, common to all three groups, performance on the $B$ discrimination was better when the inter$B$ interval was longer. (Note that the inter-B interval remained confounded with the number of $B$ discrimination
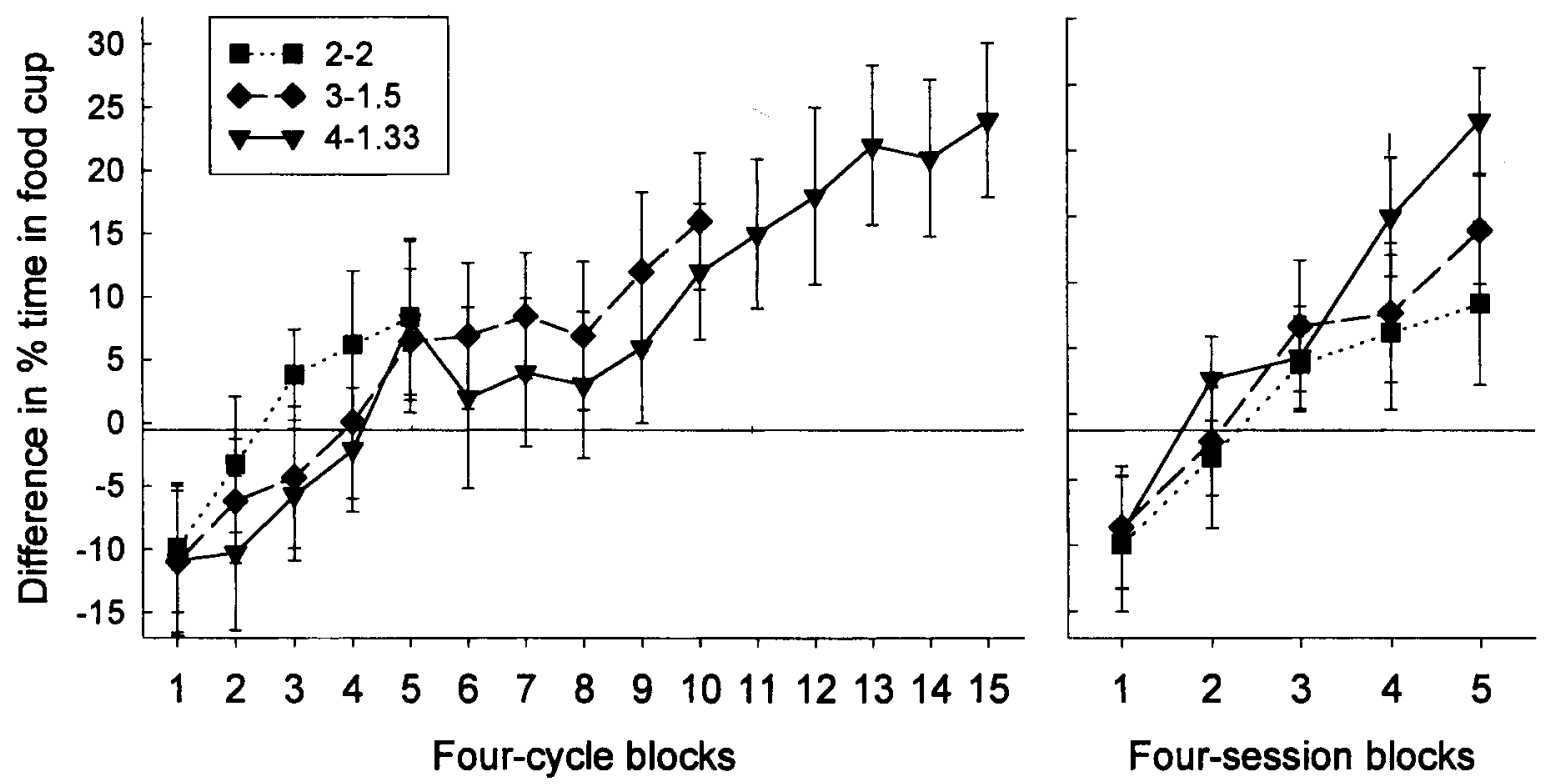

Figure 5. Acquisition (mean $\pm S E M$ ) of discriminative responding (responding on reinforced compound trials minus responding on nonreinforced target-alone trials) for the B discrimination in Experiment 3, plotted by cycles (left panel) and sessions (right panel). Each cycle comprised 1 reinforced compound trial and 3 nonreinforced target-alone trials. See text for explanation of the group designations in the legend. 
trials received in each session, as in Experiment 2, as well as the total number of training trials in each session.) This claim was supported by a statistical analysis like that reported in Experiment 2 . A group $\times$ session ANOVA of the difference scores for the B discrimination was conducted over the first five four-cycle blocks (common to all groups). This analysis showed reliable effects of group $[F(2,21)=3.89]$ and cycle blocks $[F(4,84)=188.42]$; the interaction was not reliable. Tukey HSD tests showed the discrimination difference scores to be reliably greater in Group 2-2 than in either Group 3-1.5 or Group 4-1.3, which did not differ. Finally, with extended training, performance of the two groups with shorter inter-B intervals (3-1.5 and 4-1.3) attained respectable levels.

The right side of Figure 5 shows acquisition of the B discrimination, plotted as a function of sessions. Unlike in Experiment 2, the more B discrimination trials given, the better the performance on that discrimination, despite the shorter inter-B intervals associated with more training trials. A group $\times$ session ANOVA showed a reliable effect of session $[F(4,84)=195.61]$ and a significant group $\times$ session interaction $[F(8,84)=9.71]$. It is perhaps not surprising that the $50 \%$ longer inter-B interval in Group 2-2, compared with Group 4-1.3, was overwhelmed by the threefold difference in the number of $B$ discrimination trials per session across those groups. Note that, in Experiment 2, in which there were no group effects when the data were plotted as a function of sessions, the threefold difference in the number of discrimination trials per session across conditions was balanced by a threefold difference in the discrimination-specific ITI.

Table 2 shows A discrimination responding in the test sessions in which responding of all three groups was tested with both 2-min and 4-min inter-A intervals. The overall ITI was kept constant at $1 \mathrm{~min}$ by interpolating different numbers of trials from the $B$ discrimination. Testing at the shorter inter-A interval impaired performance of the rats trained with the longest inter-A interval (Group 4-1.3), but testing with the longer interval did not enhance performance of the rats trained with the shorter inter-A intervals. A training interval (group) $\times$ test interval $\times$ test stimulus ANOVA showed reliable effects of group $[F(2,21)=5.96]$ and test stimulus $[F(3,63)=$
$85.83]$ and significant group $\times$ test stimulus $[F(6,63)=$ $8.11]$, test interval $\times$ test stimulus $[F(3,63)=25.46]$, and three-way $[F(6,63)=7.87]$ interactions. Tukey HSD tests showed that, in Group 4-1.3, test with the shorter (2-min) inter-A interval depressed responding on $\mathrm{X} \rightarrow \mathrm{A}$ trials but increased responding on $\mathrm{A}$-alone trials. There were no other reliable differences between 4-min and 2-min test responding within any group.

Finally, responding on each trial type in the test sessions was examined as a function of the identity of the immediately preceding trial type (Table 3 ). Performance was disrupted by "negative trial transitions" within a discrimination. Responding on B-alone trials was elevated when they were preceded by $\mathrm{Y} \rightarrow \mathrm{B}$ or $\mathrm{X} \rightarrow \mathrm{A}$ trials, relative to when they were preceded by $\mathrm{A}$-alone or $\mathrm{B}$-alone trials, and responding on A-alone trials was elevated when they were preceded by $\mathrm{X} \rightarrow \mathrm{A}$ or $\mathrm{Y} \rightarrow \mathrm{B}$ trials, relative to when they were preceded by A-alone or B-alone trials. Furthermore, these latter deleterious effects of compound presentations on trial $n-1$ were greater when they contained the same single element that was presented on trial $n$ (e.g., $\mathrm{X} \rightarrow \mathrm{A}$ before $\mathrm{A}$-alone). Likewise, responding to the $X \rightarrow A$ compound was lower following presentations of $A$ alone than following presentations of $B$ alone.

A group $\times$ trial $n$ type $\times$ trial $n-1$ type ANOVA for responding on $\mathrm{B}$-alone and $\mathrm{Y} \rightarrow \mathrm{B}$ trials showed reliable effects of all three factors $[F(2,21)=13.17 ; F(1,21)=$ 39.45 ; and $F(3,63)=24.25$, respectively] and a trial $n$ type $\times$ trial $n-1$ type interaction $[F(3,63)=9.92]$. Tukey HSD tests showed that responding on B-alone trials was greater after a $\mathrm{Y} \rightarrow \mathrm{B}$ trial than after any other kind of trial and was greater after an $X \rightarrow A$ trial than after an A-alone trial. Similar tests showed no differences in responding on $\mathrm{Y} \rightarrow \mathrm{B}$ trials as a function of the preceding trial. A group $\times$ trial $n-1$ type ANOVA of responding on A-alone trials showed only a reliable effect of $n-1$ trial type $[F(3,63)=18.94]$. Tukey HSD tests showed that responding on A-alone trials was greater after an $\mathrm{X} \rightarrow \mathrm{A}$ trial than after any other trial type and was greater after a $\mathrm{Y} \rightarrow \mathrm{B}$ trial than after another A-alone trial. Because there were only $2 \mathrm{X} \rightarrow \mathrm{A}$ trials in the test, only the effects of prior $\mathrm{A}$-alone and $\mathrm{B}$-alone trials on responding on $\mathrm{X} \rightarrow \mathrm{A}$ trials were examined. A group $\times$ trial $n-1$ type

Table 2

Performance Test in Experiment 3 (Percentage of Time in Food Cup)

\begin{tabular}{|c|c|c|c|c|c|c|c|c|c|c|c|c|}
\hline \multirow[b]{3}{*}{ Trial Type } & \multicolumn{4}{|c|}{ Group 2-2 } & \multicolumn{4}{|c|}{ Group 3-1.5 } & \multicolumn{4}{|c|}{ Group 4-1.3 } \\
\hline & \multicolumn{2}{|c|}{$2-2$} & \multicolumn{2}{|c|}{$4-1.3$} & \multicolumn{2}{|c|}{$2-2$} & \multicolumn{2}{|c|}{$4-1.3$} & \multicolumn{2}{|c|}{$2-2$} & \multicolumn{2}{|c|}{$4-1.3$} \\
\hline & $M$ & $S E M$ & $M$ & $S E M$ & $M$ & $S E M$ & $M$ & SEM & $M$ & $S E M$ & $M$ & $S E M$ \\
\hline$X \rightarrow A$ & 44.5 & \pm 2.5 & 42.9 & \pm 2.4 & 46.6 & \pm 2.5 & 50.2 & \pm 2.8 & 49.9 & \pm 2.8 & 58.4 & \pm 3.2 \\
\hline A & 35.8 & \pm 2.7 & 33.9 & \pm 2.4 & 36.7 & \pm 2.0 & 31.3 & \pm 2.2 & 38.2 & \pm 3.0 & 24.6 & \pm 2.7 \\
\hline $\mathrm{Y} \rightarrow \mathrm{B}$ & 44.2 & \pm 1.5 & 42.5 & \pm 1.3 & 55.1 & \pm 2.6 & 53.4 & \pm 3.1 & 66.5 & \pm 3.3 & 64.5 & \pm 3.5 \\
\hline B & 34.0 & \pm 2.6 & 33.9 & \pm 2.3 & 36.1 & \pm 1.2 & 38.2 & \pm 1.3 & 42.1 & \pm 2.0 & 44.4 & \pm 2.3 \\
\hline
\end{tabular}

Note-Entries are mean \pm SEM percentage of time in the food cup. $\mathrm{X}=$ feature from the A discrimination; $A=$ target from the $A$ discrimination; $Y=$ feature from the $B$ discrimination; $B=$ target from the $B$ discrimination. 2-2 refers to the tests in which both inter-A and inter-B intervals were $2 \mathrm{~min} ; 4-1.3$ refers to the tests in which inter$A$ interval was $4 \mathrm{~min}$ and inter-B interval was $1.3 \mathrm{~min}$. 
Table 3

Sequential Analysis of Experiment 3 Test Data (Percentage of Time in Food Cup)

\begin{tabular}{|c|c|c|c|c|c|c|c|c|}
\hline \multirow[b]{4}{*}{ Trial $n-1$ Type } & \multicolumn{4}{|c|}{ 2-2 Test } & \multicolumn{4}{|c|}{ 4-1.3 Test } \\
\hline & \multicolumn{4}{|c|}{ Trial $n$ type } & \multicolumn{4}{|c|}{ Trial $n$ Type } \\
\hline & \multicolumn{2}{|c|}{$X \rightarrow A$} & \multicolumn{2}{|c|}{ A } & \multicolumn{2}{|c|}{$\mathrm{Y} \rightarrow \mathrm{B}$} & \multicolumn{2}{|c|}{ B } \\
\hline & $M$ & $S E M$ & $M$ & $S E M$ & $M$ & $S E M$ & $M$ & $S E \Lambda$ \\
\hline$X \rightarrow A$ & & & 43.3 & \pm 2.0 & 53.1 & \pm 2.8 & 41.0 & \pm 1 \\
\hline A & 44.4 & \pm 1.5 & 29.2 & \pm 2.1 & 53.8 & \pm 2.5 & 34.6 & \pm 2 \\
\hline $\mathrm{Y} \rightarrow \mathrm{B}$ & & & 34.9 & \pm 1.7 & 55.3 & \pm 2.7 & 50.9 & \pm 2 \\
\hline $\mathrm{B}$ & 49.6 & \pm 2.6 & 32.1 & \pm 2.1 & 49.6 & \pm 2.1 & 35.3 & \pm 1 \\
\hline
\end{tabular}

Note-Entries are mean $( \pm S E M)$ percentage of time in the food cup, collapsed across all groups. $X=$ feature from the A discrimination; $A=$ target from the A discrimination; $Y=$ feature from the $B$ discrimination; $\mathrm{B}=$ target from the $\mathrm{B}$ discrimination. $2-2$ refers to the tests in which both inter-A and inter-B intervals were $2 \mathrm{~min} ; 4-1.3$ refers to the tests in which inter-A interval was $4 \mathrm{~min}$ and inter-B interval was $1.3 \mathrm{~min}$.

ANOVA of $\mathrm{X} \rightarrow \mathrm{A}$ responding also showed only a reliable effect of $n-1$ trial type $[F(1,21)=7.41]$; responding on $X \rightarrow A$ trials was depressed when they were preceded by $A$ alone, relative to when they were preceded by $B$ alone.

The observation of interference with responding on A-alone trials by prior $\mathrm{Y} \rightarrow \mathrm{B}$ trials and of responding on $\mathrm{B}$-alone trials by prior $\mathrm{X} \rightarrow \mathrm{A}$ trials might indicate that the attempt to minimize generalization between the $A$ and $B$ discriminations was not entirely successful. Alternately, sucrose delivery, common to both types of compound trials, may have contributed to the interference effect.

\section{GENERAL DISCUSSION}

Consistent with Holland's (1995) findings with operant conditioning procedures, feature positive discrimination learning using Pavlovian appetitive conditioning procedures was more rapid with longer ITIs (Experiments 1 and 2). Also as in Holland's (1995) study, in Experiment 1, there was less transfer of the feature's behavioral control to a separately trained target cue after training with longer ITIs, suggesting that longer ITIs encouraged the use of an occasion setting strategy. Finally, the results of Experiments 2 and 3 go beyond Holland's (1995) data in suggesting that both the overall ITI and the interval between trials relevant to a particular discrimination play roles in determining performance. Learning and performance on one feature positive discrimination was depressed less when the overall ITI was reduced by interspersing trials from a second feature positive discrimination than when it was reduced by providing additional trials from the first discrimination.

A variety of accounts have been offered for spaced practice effects. For example, some authors (e.g., Mustaca, Gabelli, Papini, \& Balsam, 1991) have suggested that shorter ITIs and, hence, higher rates of US presentation encourage greater context conditioning, which might in turn influence the effectiveness of various trial types in generating discrimination learning or performance. In the present experiments, the rats trained with lower rates of US presentation (longer overall ITIs) showed faster discrimination learning than did those trained with higher rates. However, in Experiments 2 and 3, groups of rats trained with identical US presentation rates showed different rates of discrimination learning, depending on whether the additional USs were presented with the target cue from the primary discrimination or the target from a second discrimination.

Although it seems unlikely that the use of two discriminations rather than one would substantially affect context conditioning in these experiments, there is theoretical precedent for the suggestion that intermixing two types of reinforced trials might alter the influence of context conditioning on performance to an explicit CS. In the service of accounting for the differential effects of intermixing signaled and unsignaled US presentations on conditioning, Cooper, Aronson, Balsam, and Gibbon (1990) outlined a "deletion comparator" extension of Gibbon and Balsam's application of scalar expectancy theory (Gibbon, 1977) to conditioning. In Gibbon and Balsam's account, response performance to a CS reflects the ratio of the average delay to US delivery during the session as a whole to the average delay to US delivery during the CS (the cycle/trial, or $\mathrm{C} / \mathrm{T}$, ratio). Longer ITIs generate greater performance by increasing the cycle time. Cooper et al. proposed that when there were multiple CSs, USs signaled with one CS (and the duration of that CS) were somehow deleted from the comparison term (the deletioncycle duration, or $C_{D}$ ). If an analogous deletion occurred in the present experiments, the CSs and USs from the B discrimination would be omitted from the comparison process that determines performance on A discrimination trials, leaving only the additional empty intervals in the calculation. Thus, A discrimination performance should be better with longer inter-A intervals even when those intervals include trials from another discrimination, consistent with the data of Experiments 2 and 3. Furthermore, because this deletion calculation would exclude the durations of the CSs from the B discrimination, inserting $B$ trials when the inter- $A$ interval is extended would produce poorer performance than merely extending the ITI. This expectation was also confirmed in Experiment 2: Acquisition was more rapid in Group 4-4, in which the 4-min inter-A intervals were empty, than in Group 4-1.3, in which those intervals included trials from the B discrimination. Applicability of this approach to the present data, however, awaits further explication of the nature of such a deletion mechanism.

Another approach to understanding the data of these experiments follows the lead of Kehoe, Cool, and Gormezano (1991), who suggested that longer ITIs enhance discrimination learning by reducing proactive interference from preceding trials (see Wright, Urcuioli, \& Sands, 1986, for a review). Many investigators have found trial sequence effects on learning and performance on discrimination tasks, such that performance on a given trial is degraded if the immediately preceding trial was of the 
opposite significance (e.g., Grant, 1975; Hogan et al., 1981; Pontecorvo, 1983) or generated a response that was incorrect for the present trial (e.g., Roitblat \& Scopatz, 1983). Learning or performance would be impaired to the extent that a memorial record of a previous trial interfered with processing of the current trial or disrupted the proper assignment of the effects of reinforcement or nonreinforcement to the cues present on the current trial.

Generally, these deleterious effects attributed to proactive interference are greater the shorter the ITI (e.g., Grant, 1975; Roitblat \& Scopatz, 1983); thus longer ITIs would support more rapid learning. Furthermore, it is commonly assumed that interference among similar items is greater than that among dissimilar items (e.g., Niemark, Greenhouse, Law, \& Weinheimer, 1965). As a result, in the present experiments, interpolation of trials from the B discrimination might produce less interference with performance on the A discrimination than interpolation of additional A trials. For example, the effects of an $\mathrm{X}-\mathrm{A}+$ trial might be reduced more by an immediately preceding $\mathrm{A}-$ trial than by a $\mathrm{B}-$ trial. Consequently, both the overall ITI and the inter-A interval would be expected to influence discrimination performance. The results of the test session of Experiment 3 supported this analysis: Responding on A - trials was less accurate (a greater percentage of time was spent in the food cup) after $\mathrm{X}-\mathrm{A}+$ trials than after $\mathrm{Y}-\mathrm{B}+$ trials.

Using an operant "ambiguous feature" discrimination procedure $(\mathrm{X} \rightarrow \mathrm{A}+/ \mathrm{A}-/ \mathrm{X} \rightarrow \mathrm{B}-/ \mathrm{B}+)$ related to the feature positive discriminations used here, Han et al. (1998) also noted an interaction between ITI and trial sequence that was consistent with this proactive interference account of ITI effects. With short (0.5- or 1-min) ITIs, they found poorer discrimination performance on A-alone trials when they were preceded by $\mathrm{X} \rightarrow \mathrm{A}$ trials than when they were preceded by any other kind of trial, and they found poorer performance on $\mathrm{X}-\mathrm{B}$ trials when they were preceded by $B$-alone trials than when they were preceded by any other type of trial. In contrast, with long (8-min) ITIs in testing, trial sequence was irrelevant.

Interestingly, Han et al. (1998) found that rats with hippocampal lesions failed to show these sequential effects when trained and tested with short ITIs, and, indeed, these rats showed more accurate performance overall than did normal rats. These results led Han et al. to speculate that the lesioned rats had working memory deficits such that the record of the previous trial faded rapidly, relative to normal rats. As a result, lesioned rats would not be subject to proactive interference effects even with short ITIs and, hence, might display superior performance with short ITIs. By contrast, when trained and tested with long ITIs, performance of lesioned rats and normal rats did not differ. Both groups of rats showed comparable discrimination accuracy, and neither showed evidence for sequence effects, consistent with the expectation that long ITIs would minimize the importance of proactive interference effects. To the extent that the superiority of the performance of rats in the groups with interpolated $B$ trials to those with additional $A$ trials resulted from similar proactive interference effects, one would predict that, although rats with hippocampal lesions would show better performance than normal rats when trained with short inter-A intervals, that advantage would be reduced when the inter- $A$ interval was extended (but the overall ITI was left unchanged) by the interpolation of trials from a second, B, discrimination.

Although the differential effects of interpolating A and B discrimination trials may be well described by this proactive interference account, other evidence from Han et al.'s (1998) study suggests that proactive interference alone is not likely to be responsible for the advantage of longer overall ITIs. If the lesioned rats were unable to hold a record of the preceding trial for as long as $30 \mathrm{sec}$, as suggested by their failure to show sequence effects, then there would be little difference in the amount of proactive interference regardless of whether the training ITI was $1 \mathrm{~min}$ or $8 \mathrm{~min}$. But, like normal rats, lesioned rats showed much more rapid acquisition of the ambiguous discrimination with 8-min ITIs than with 1-min ITIs. Clarification of the mechanisms by which ITI affects feature positive discrimination performance awaits further study.

\section{REFERENCES}

Cooper, L. D., Aronson, L., Balsam, P. D., \& Gibbon, J. (1990). Duration of signals for intertrial reinforcement and nonreinforcement in random control procedures. Journal of Experimental Psychology: Animal Behavior Processes, 16, 14-26.

GibBon, J. (1977). Scalar expectancy theory and Weber's law in animal timing. Psychological Review, 84, 279-325.

Gibbon, J., Baldock, M. D., Locurto, C. M., Gold, L., \& Terrace, H. S. (1977). Trial and intertrial durations in autoshaping. Journal of Experimental Psychology: Animal Behavior Processes, 3, 264-284.

GibBON, J., \& BALSAM, P. (1981). Spreading association in time. In C. M. Locurto, H. S. Terrace, \& J. Gibbon (Eds.), Autoshaping and conditioning theory (pp. 219-253). New York: Academic Press.

Gormezano, I., \& MoORE, J. W. (1969). Classical conditioning. In M. H. Marx (Ed.), Learning: Processes (pp. 121-203). London: MacMillan.

Grant, D. S. (1975). Proactive interference in pigeon short-term memory. Journal of Experimental Psychology: Animal Behavior Processes, 1 , 207-220.

Han, J. S., Gallagher, M., \& Holland, P. C. (1998). Hippocampal lesions enhance configural learning by reducing proactive interference. Hippocampus, 8, 138-146.

Hogan, D. E., Edwards, C. A., \& Zentall, T. R. (1981). Delayed matching in the pigeon: Interference produced by the prior delayed matching trial. Animal Learning \& Behavior, 9, 395-400.

Holland, P. C. (1977). Conditioned stimulus as a determinant of the form of the Pavlovian conditioned response. Journal of Experimental Psychology: Animal Behavior Processes, 3, 77-104.

Holland, P. C. (1989a). Feature extinction enhances transfer of occasion setting. Animal Learning \& Behavior, 17, 269-279.

Holland, P. C. (1989b). Transfer of negative occasion setting and conditioned inhibition across conditioned and unconditioned stimuli. Journal of Experimental Psychology: Animal Behavior Processes, 15, 311-328.

Holland, P. C. (1992). Occasion setting in Pavlovian conditioning. In D. Medin (Ed.), The psychology of learning and motivation (Vol. 28, pp. 69-125). San Diego: Academic Press.

Holland, P. C. (1995). The effects of intertrial interval (ITI) on learn- 
ing and performance of serial feature positive discriminations. Animal Learning \& Behavior, 23, 411-428.

Kehoe, E. J., COOL, V., \& GoRmezano, I. (1991). Trace conditioning of the rabbit's nictitating membrane response as a function of CS-US interstimulus interval and trials per session. Learning \& Motivation, 22, 269-290.

Mustaca, A. E., Gabelli, F., Papini, M. R., \& Balsam, P. (1991). The effects of varying the interreinforcement interval on appetitive contextual conditioning. Animal Learning \& Behavior, 19, 125-138.

Niemark, E. D., Greenhouse, P., LAW, S., \& Weinheimer, S. (1965). The effect of rehearsal preventing tasks upon retention of CVC syllables. Journal of Verbal Learning \& Verbal Behavior, 4, 280-285.

PAPINI, M. R., \& DUDLEY, R. T. (1993). Effects of the number of trials per session on autoshaping in rats. Learning \& Motivation, 24, 175-193.

PonTeCorvo, M. J. (1983). Effects of proactive interference on rats' continuous nonmatching-to-sample performance. Animal Learning \& Behavior, 11, 356-366.
Roberts, W. A., \& KraEMER, P. J. (1982). Some observations of the effects of intertrial interval and delay on delayed matching to sample in pigeons. Journal of Experimental Psychology, 8, 342-353.

Roitblat, H. L., \& Scopatz, R. A. (1983). Sequential effects in pigeon delayed matching-to-sample performance. Journal of Experimental Psychology: Animal Behavior Processes, 9, 202-221.

SPENCE, K. W., \& NoRrus, E. B. (1950). Eyelid conditioning as a function of the inter-trial interval. Journal of Experimental Psychology, 40, 716-720.

Wright, A. A., Urcuioli, P. J., \& Sands, S. F. (1986). Proactive interference in animal memory. In D. F. Kendrick, M. E. Rilling, \& M. R. Denny (Eds.), Theories of animal memory (pp. 101-125). Hillsdale, NJ: Erlbaum.

(Manuscript received July 29, 1998; revision accepted for publication January $7,1999$. 